\title{
THE CONCORDANCE CLASSIFICATION OF LOW CROSSING NUMBER KNOTS
}

\author{
JULIA COLLINS, PAUL KIRK, AND CHARLES LIVINGSTON
}

(Communicated by Daniel Ruberman)

\begin{abstract}
We present the complete classification of the subgroup of the classical knot concordance group generated by prime knots with eight or fewer crossings. Proofs are presented in summary. We also describe extensions of this work to the case of nine crossing knots.
\end{abstract}

\section{INTRODUCTION}

Recall that knots $K$ and $J$ in $S^{3}$ are called concordant if there is a smooth embedded disk in $B^{4}$ with boundary $K \#-J$. The set of equivalence classes under this relation forms the smooth concordance group $\mathcal{C}$, an abelian group with addition induced by connected sums. Initial work by Fox and Milnor [9] and Musasugi [23] developed obstructions that were sufficient to prove that the figure eight knot, $4_{1}$, represents a nontrivial element of order two in $\mathcal{C}$ and that the trefoil, $3_{1}$, is of infinite order.

The analysis of the information carried by the universal abelian cover of a knot complement (called the algebraic information) culminated with Levine's definition [18] of a homomorphism $\phi$ from $\mathcal{C}$ onto the algebraic concordance group $\mathcal{G}$, a group isomorphic to $\mathbf{Z}_{2}^{\infty} \oplus \mathbf{Z}_{4}^{\infty} \oplus \mathbf{Z}^{\infty}$. The nontriviality of the kernel of $\phi$ was proved by Casson and Gordon [1].

Since then, techniques of increasing effectiveness have been developed to study $\mathcal{C}$. For the most part, these have been applied to consider individual knots or to study specified families of knots, for instance, two-bridge knots [1], pretzel knots [8, 12, positive knots [25, and Whitehead doubles [4, 5, 13]. Beyond these examples, throughout the study of concordance investigators have built families of knots specifically designed to realize newly discovered invariants; two examples among many are 6, 11]. Despite these many advances, the classification problem for $\mathcal{C}$ remains far out of reach.

Our goal is to approach the general classification by completing the classification for the subgroup generated by prime knots of eight or fewer crossings. In doing so, we will illustrate the effectiveness of twisted Alexander polynomials to resolve previously unmanageable examples of potential concordances. In the appendix we will summarize results of the first author [3] for the much larger group generated by prime knots of nine and fewer crossing knots.

Received by the editors November 19, 2013 and, in revised form, June 15, 2014.

2010 Mathematics Subject Classification. Primary 57M25; Secondary 57N70, 57Q45.

This work was supported in part by the National Science Foundation under Grant 1007196, and by Simons Foundation Grants 278714 and 209082. 
One might ask precisely what is meant by classification. From the perspective of fully understanding knot concordance, at the very least this needs to include an algorithm to determine if a given linear combination of low crossing number knots is trivial. But in addition, the classification should identify the order of an element and its value in the algebraic concordance group $\mathcal{G}$ under Levine's homomorphism $\phi$. Here is a summary statement. The full result, filling in details concerning orientation issues, will be provided in the next section.

Theorem 1. There are 36 oriented prime knots of eight or fewer crossings. These knots generate a subgroup $\mathcal{C}_{8} \subset \mathcal{C}$ isomorphic to $\left(\mathbf{Z}^{18} \oplus \mathbf{Z}_{2}^{6}\right) \oplus\left(\mathbf{Z}^{5} \oplus \mathbf{Z}_{2}\right)$. The first of the two summands, $\left(\mathbf{Z}^{18} \oplus \mathbf{Z}_{2}^{6}\right)$, maps injectively into $\mathcal{G}$; for the second summand, the $\mathbf{Z}^{5}$ has image in $\mathcal{G}$ isomorphic to $\mathbf{Z}_{4} \oplus \mathbf{Z}_{2}^{3}$ and the $\mathbf{Z}_{2}$ maps trivially to $\mathcal{G}$.

The proof of this theorem is such that determining the concordance class of any linear combination of prime knots, each of eight or fewer crossings, is completely algorithmic. In fact, an online calculator developed by the first author, available at KnotInfo [2], permits one to easily determine the concordance properties, including the image in the algebraic concordance group $\mathcal{G}$, of any given linear combination of prime knots with eight or fewer crossings.

1.1. Topological concordance. The topological concordance group $\mathcal{C}_{\text {top }}$ is defined via topologically locally flat concordances rather than smooth concordances. There is a surjection $\mathcal{C} \rightarrow \mathcal{C}_{\text {top }}$; the kernel is nontrivial, including all knots of Alexander polynomial 1 [10. The methods of this paper all apply in the topological setting, and thus Theorem 1 holds with smooth replaced by topological concordance.

Perhaps the first distinction between the two categories occurs with the knot $11_{31}$, which has smooth four-genus 2 ; it has Rasmussen invariant, as defined in [24], $s\left(11_{31}\right)=-4$, and in general, $2 g_{4}(K) \geq|s(K)|$. On the other hand, Stoimenow constructed a genus one cobordism to an Alexander polynomial one knot, and thus $11_{31}$ has topological four-genus at most 1 . Its classical signature is -2 , and thus its topological four-genus is exactly 1.

1.2. Classification of nine crossing knots. The concordance classification of nine crossing knots is not complete. In the Appendix we discuss progress made by the first author in classifying the subgroup generated by prime knots of nine or fewer crossings. That classification awaits further work before publication. The remaining unknown cases provide a provocative set of examples for further investigation, as summarized with the following problem.

Problem. The five knots $2\left(9_{2}-7_{4}\right), 8_{21}-8_{18}-3_{1}, 9_{23}-9_{2}-3_{1}, 9_{32}^{r}-9_{32}$, and $9_{40}-818-4_{1}-3_{1}$ are each algebraically slice and of infinite order in $\mathcal{C}$. Are they linearly independent in $\mathcal{C}$ ? Are they linearly independent in $\mathcal{C}_{\text {top }}$ ?

There is little evidence as to whether these knots are independent, and thus we make no conjecture. The unresolved cases might provide examples in which smooth and topological concordance differ, but as of yet, no known obstructions have succeeded with these knots. In the case that they are independent, the following would correspond to Theorem 1 ,

Possible concordance classification. There are 87 oriented prime knots of nine or fewer crossings. These knots generate a subgroup $\mathcal{C}_{9} \subset \mathcal{C}$ isomorphic to $\left(\mathbf{Z}^{46} \oplus\right.$ $\left.\mathbf{Z}_{2}^{6}\right) \oplus\left(\mathbf{Z}^{23} \oplus \mathbf{Z}_{2}\right)$. The first of the two summands, $\left(\mathbf{Z}^{46} \oplus \mathbf{Z}_{2}^{6}\right)$, maps injectively into 
$\mathcal{G}$; for the second summand, the $\mathbf{Z}^{23}$ has image in $\mathcal{G}$ isomorphic to $\mathbf{Z}_{4}^{2} \oplus \mathbf{Z}_{2}^{13}$ and the $\mathbf{Z}_{2}$ maps trivially to $\mathcal{G}$.

\section{Statement of Classification}

There are 36 prime knots of eight or fewer crossings; every knot of eight or fewer crossings can be expressed as a connected sum of these prime knots. In this count, we include only one knot from each pair $\{K$, mirror $(K)\}$ for those knots $K$ which are reversible, since the mirror image with its orientation reversed represents $-K$ in $\mathcal{C}$. However, there is one nonreversible knot, $8_{17}$, so for completeness we must include $8_{17}$ and $8_{17}^{r}$ in the count. Thus, the set of knots we consider is

$$
3_{1}, 4_{1}, 5_{\{1-2\}}, 6_{\{1-3\}}, 7_{\{1-7\}}, 8_{\{1-21\}}, 8_{17}^{r} .
$$

Let $\mathcal{F}$ denote the free abelian group generated by these 36 knots. Levine provided a classification result for $\mathcal{G}$ based on a set of explicit invariants (enhanced slightly in [19,21] to consider four torsion). Using this, an initial decomposition becomes available.

Lemma 2. There is a direct sum decomposition $\mathcal{F}=\mathcal{F}_{\infty} \oplus \mathcal{F}_{4} \oplus \mathcal{F}_{2} \oplus \mathcal{F}_{1}$, with summands of rank $18,1,9$, and 8 , respectively. The group $\mathcal{F}_{\infty}$ maps to a summand of $\mathcal{G}$ isomorphic to $\mathbf{Z}^{18} ; \mathcal{F}_{4}$ and $\mathcal{F}_{2}$ map onto complementary summands of $\mathcal{G}$ isomorphic to $\mathbf{Z}_{4}$ and $\mathbf{Z}_{2}^{9}$, respectively; $\mathcal{F}_{1}$ maps trivially to $\mathcal{G}$.

The next three results will be seen to be consequences of Casson-Gordon theory.

Lemma 3. The group $\mathcal{F}_{4} \cong \mathbf{Z}$ injects into $\mathcal{C}$.

Lemma 4. The group $\mathcal{F}_{2}$ has a further decomposition as $\mathcal{F}_{2}^{\infty} \oplus \mathcal{F}_{2}^{2}$, of ranks 3 and 6 , respectively. The group $\mathcal{F}_{2}^{\infty}$ maps onto $\mathbf{Z}^{3} \subset \mathcal{C}$ and $\mathcal{F}_{2}^{2}$ maps surjectively to $\mathbf{Z}_{2}^{6} \subset \mathcal{C}$.

Lemma 5. The group $\mathcal{F}_{1}$ has a further decomposition as $\mathcal{F}_{1}^{\infty} \oplus \mathcal{F}_{1}^{2} \oplus \mathcal{F}_{1}^{1}$, of ranks 1,1 and 6 , respectively. The group $\mathcal{F}_{1}^{\infty}$ maps isomorphically onto $\mathbf{Z} \subset \mathcal{C}, \mathcal{F}_{1}^{2}$ maps onto $\mathbf{Z}_{2} \subset \mathcal{C}$, and $\mathcal{F}_{1}^{1}$ maps trivially to $\mathcal{C}$.

With these groups defined, the classification is essentially completed by describing the bases of each of these summands.

Theorem 6. Bases for the subgroups described in the previous lemmas are given in the following list. (For clarity and consistency we write $\mathcal{F}_{\infty}^{\infty}$ for $\mathcal{F}_{\infty}$ and $\mathcal{F}_{4}^{\infty}$ for $\mathcal{F}_{4}$. In this way, subscripts indicate orders in $\mathcal{G}$ and superscripts give the order in C.)

- $\mathcal{F}_{\infty}^{\infty}: 3_{1}, 5_{\{1,2\}}, 6_{2}, 7_{\{1-6\}}, 8_{\{2,4,5,6,7,14,16,19\}}$

- $\mathcal{F}_{4}^{\infty}: 7_{7}$

- $\mathcal{F}_{2}^{\infty}: 8_{1}, 8_{13},\left(8_{15}-7_{2}-3_{1}\right)$

- $\mathcal{F}_{2}^{2}: 4_{1}, 6_{3}, 8_{\{3,12,17,18\}}$

- $\mathcal{F}_{1}^{\infty}:\left(8_{21}-8_{18}-3_{1}\right)$

- $\mathcal{F}_{1}^{2}:\left(8_{17}-8_{17}^{r}\right)$

- $\mathcal{F}_{1}^{1}: 6_{1}, 8_{\{8,9,20\}},\left(8_{10}+3_{1}\right),\left(8_{11}-3_{1}\right)$ 


\section{EXAmples}

Two examples illustrate how the previous theorem provides a complete classification.

3.1. $K=8_{10} \# 8_{21}$. Consider the knot $K=8_{10} \# 8_{21}$. Writing $K$ in terms of the bases given above, we have

$$
K=\left(8_{10} \# 3_{1}\right) \#\left(8_{21} \#-8_{18} \#-3_{1}\right) \# 8_{18},
$$

the sum of generators of $\mathcal{F}_{1}^{1}, \mathcal{F}_{1}^{\infty}$ and $\mathcal{F}_{2}^{2}$. Thus, $K$ maps to an element of order two in $\mathcal{G}$ and represents an element of infinite order in $\mathcal{C}$.

Remark. Both $8_{10}$ and $8_{21}$ have nontrivial signature functions, identical to those of $3_{1}$ and $-3_{1}$, and so individually have infinite order in $\mathcal{C}$. However, the sum has trivial signature function, and thus it represents a torsion element in $\mathcal{G}$. Their Alexander polynomials are the same as those of $3_{1} \# 3_{1} \# 3_{1}$ and $3_{1} \# 4_{1}$, respectively. Thus, $8_{10}$ and $8_{21}$ are distinct in $\mathcal{C}$, since the product of their Alexander polynomials is not of the form $f(t) f\left(t^{-1}\right)$ for some $f$ (the Fox-Milnor [9] obstruction to being slice). Proving that this knot is of infinite order in $\mathcal{C}$ is more challenging; the only proof we know depends on a careful analysis of branched covers and twisted Alexander polynomials.

3.2. $K=8_{17}^{r} \# 8_{21} \#-3_{1}$. In this case, we rewrite $K$ as

$$
K=-\left(8_{17}-8_{17}^{r}\right) \# 8_{17} \#\left(8_{21} \#-8_{18} \#-3_{1}\right) \# 8_{18} .
$$

The four knots in this decomposition are in $\mathcal{F}_{1}^{2}, \mathcal{F}_{2}^{2}, \mathcal{F}_{1}^{\infty}$, and $\mathcal{F}_{2}^{2}$ respectively. From this it follows that $K$ is of algebraic order two, but is of infinite order in concordance.

3.3. Calculator. As these examples illustrate, the problem of determining the (algebraic) concordance class of a knot and its order has been reduced to simple linear algebra. A program, accessible via the Internet, has been written to implement the algorithm. The reader is invited to visit the "Concordance Calculator" posted on KnotInfo 2] to generate more examples.

\section{Algebraic classification}

The definitions of $\mathcal{F}_{\infty}, \mathcal{F}_{4}, \mathcal{F}_{2}$, and $\mathcal{F}_{1}$ are algebraic, depending only on the image of the sets of knots in the algebraic concordance group $\mathcal{G}$. Levine's classification of $\mathcal{G}$ is for the most part algorithmic and thus the problem of identifying the image of $\mathcal{F}$ in $\mathcal{G}$ follows from his work.

We recall briefly one of the key features of Levine's classification. The group $\mathcal{G}$ is defined as certain equivalence classes of Seifert matrices $V_{K}$ with block sum as the addition. For any field $\mathbf{F}$ containing $\mathbf{Q}$, one defines a group $\mathcal{G}_{\mathbf{F}}$ which $\mathcal{G}$ maps onto. For $\mathbf{F}=\mathbf{Q}, \mathcal{G}_{\mathbf{F}}=\mathcal{G}$. There is a decomposition $\mathcal{G}_{\mathbf{F}} \cong \oplus_{f(t)} \mathcal{G}_{f(t)}$ where the $f(t)$ are taken from the set of distinct $\mathbf{F}$-irreducible symmetric Alexander polynomials. A knot maps to summands corresponding to factors of its Alexander polynomial.

For $\mathbf{F}=\mathbf{R}$, the real numbers, the relevant polynomials $f$ are irreducible quadratics having roots on the unit circle. Each of the corresponding summands is isomorphic to Z . To a knot $K$ with Seifert matrix $V_{K}$ and a polynomial $f$ having its roots at $e^{ \pm 2 \pi i \theta}$, one assigns the integer which is the jump in the signature function

$$
\sigma_{\omega}(K)=\sigma_{\omega}\left(V_{K}\right)=\text { signature }\left((1-\omega) V_{K}+(1-\bar{\omega}) V_{K}^{t}\right)
$$


as $\omega$ moves through $e^{2 \pi i \theta}$ on the unit circle. The intersection of the kernels of the collection of these integer valued invariants over all such quadratics $f$ is precisely the torsion in $\mathcal{G}$. This kernel can also be described as the subgroup of $\mathcal{G}$ generated by those $V_{K}$ so that $\sigma_{\omega}\left(V_{K}\right)$ is zero for all but finitely many $\omega$. The full set of invariants that detect the two and four torsion arise from considering the fields $\mathbf{F}=\mathbf{Q}_{(p)}$, the $p$-adic completions of $\mathbf{Q}$. For low crossing number knots, this $p$-adic analysis can largely be avoided.

4.1. Slice knots: $\mathcal{F}_{1}^{1}$. The first step in producing the decomposition of Theorem [6 is to isolate the known slice knots, that is, knots that represent 0 in $\mathcal{C}$. The first four elements of $\mathcal{F}_{1}^{1}$ can be quickly seen to be slice. The triviality of $8_{10} \# 3_{1}$ and $8_{21} \#-3_{1}$ in $\mathcal{C}$ was observed by Conway in [7]. It is a consequence of the results described here that there are no other such relations.

4.2. Infinite order elements and the signature function: $\mathcal{F}_{\infty}^{\infty}$. Since jumps of the signature function occur only at roots of the Alexander polynomial, for any finite set of knots, one can determine the image of the signature function by evaluating it at a set of points that includes a number on the unit circle between any two such roots of any of the Alexander polynomials that arise. For the current calculation and that for nine crossing knots, there are only 70 roots of Alexander polynomials on the upper unit half circle. Hence, evaluating the signature function at the midpoints of the circular segments joining those roots gives a homomorphism from $\mathcal{F}$ to $\mathbf{Z}^{70}$ whose kernel maps by $\phi$ to torsion in $\mathcal{G}$.

It is now an exercise in elementary linear algebra to identify the set $\mathcal{F}_{\infty}^{\infty}$ as mapping to a generating set of the free part of the image of $\mathcal{C}$ in $\mathcal{G}$ and that the remaining summands map to torsion in $\mathcal{G}$.

4.3. Torsion in $\mathcal{G}$. We now describe the image of the subgroup generated by $\mathcal{F}_{4}^{\infty}, \mathcal{F}_{2}^{\infty}, \mathcal{F}_{2}^{2}, \mathcal{F}_{1}^{\infty}$ and $\mathcal{F}_{1}^{2}$ in the torsion of $\mathcal{G}$. Levine's paper [18] provided a complete set of invariants of algebraic concordance. These included invariants arising in $\mathcal{G}_{\mathbf{Q}_{(p)}}$ for all $p$-adic completions $\mathbf{Q}_{(p)}$ of $\mathbf{Q}$, but in 21] it was shown how to reduce the set of primes to a finite collection. In [19] there was a further reduction, leading to effective means of determining the algebraic order of a knot. The knots we are considering fall to those techniques, so we only summarize the required work here.

4.3.1. $\mathcal{F}_{1}^{2}, \mathcal{F}_{1}^{\infty}$. We most quickly dispense with $\mathcal{F}_{1}^{2}$ and $\mathcal{F}_{1}^{\infty}$. Since a knot and its reverse represent the same element in $\mathcal{G}, 8_{17}-8_{17}^{r}$ is algebraically slice, that is, maps to zero in $\mathcal{G}$. (One proof of this follows from a theorem of Long [22]. For any knot $K$, the knot $K \#-K^{r}$ is "positive amphicheiral", meaning there is an orientation reversing involution of $S^{3}$ inducing an orientation preserving homeomorphism of $K \#-K^{r}$. According to 22, such knots are algebraically slice.) The analysis for $8_{21}-8_{18}-3_{1}$ is a bit more detailed. However, the Alexander polynomials of $8_{21}, 8_{18}$, and $3_{1}$ factor into irreducible quadratics, and for these Levine's criteria simplify, as described in [18, from which one can readily show that $8_{21}-8_{18}-3_{1}$ is algebraically slice; more details are presented in Section 6 .

4.3.2. $\mathcal{F}_{4}^{\infty}, \mathcal{F}_{2}^{\infty}, \mathcal{F}_{2}^{2}$. The group $\mathcal{G}$ splits as a direct sum $\oplus_{p(t)} \mathcal{G}_{p(t)}$ where the $p(t)$ are distinct irreducible symmetric rational polynomials; a knot maps to summands 
corresponding to factors of its Alexander polynomial. Here is a list of the Alexander polynomials that arise.

$$
\begin{aligned}
& \text { - } 7_{7}: 1-5 t+9 t^{2}-5 t^{3}+t^{4} \\
& \text { - } 8_{1}: 3-7 t+3 t^{2} \\
& \text { - } 8_{13}: 2-7 t+11 t^{2}-7 t^{2}+2 t^{4} \\
& \text { - } 8_{15}-7_{2}-3_{1}:\left(1-t+t^{2}\right)^{2}\left(3-5 t+3 t^{2}\right)^{2} \\
& \text { - } 4_{1}: 1-3 t+t^{2} \\
& \text { - } 6_{3}: 1-3 t+5 t^{2}-3 t^{3}+t^{4} \\
& \text { - } 8_{3}: 4-9 t+4 t^{2} \\
& \text { - } 8_{12}: 1-7 t+13 t^{2}-7 t^{3}+t^{4} \\
& \text { - } 8_{17}: 1-4 t+8 t^{2}-11 t^{3}+8 t^{4}-4 t^{5}+t^{6} \\
& \text { - } 8_{18}: 1-5 t+10 t^{2}-13 t^{3}+10 t^{4}-5 t^{5}+t^{6}
\end{aligned}
$$

Since each of these knots maps to a distinct summand of $\mathcal{G}$, we need only consider the order of the image of each individually. A few quick observations simplify the work. First, with the exception of $8_{15}-7_{2}-3_{1}$, all the polynomials are irreducible, and in particular none are of the form $f(t) f\left(t^{-1}\right)$, so each maps nontrivially to $\mathcal{G}$. Second, the knots in $\mathcal{F}_{2}^{2}$ are all (negative) amphicheiral and so are of order at most two in $\mathcal{C}$, and since they map nontrivially to $\mathcal{G}$, they have order two in $\mathcal{C}$ as well.

It remains to consider the first four knots on the list. According to Levine (see 18 for details), four torsion is detected by primes congruent to $3 \bmod 4$ dividing $|\Delta(-1)|$. The precise conditions immediately show that $7_{7}$ is of order four, while $8_{1}$ and $8_{13}$ are of order two in $\mathcal{G}$. The last case, the knot $8_{15}-7_{2}-3_{1}$, is the most subtle. Knots with these Alexander polynomials can represent four torsion in $\mathcal{G}$, but since the polynomial exponents for this knot are even, the knot must map either trivially or to two torsion. The nontriviality is detected working with the prime 11. In Section 6 more details will be presented.

\section{Algebraically slice knots and twisted Alexander polynomials}

It remains to understand the algebraically slice knots represented by elements in $\mathcal{F}$. The subgroup of such knots is generated by $4 \mathcal{F}_{4}, 2 \mathcal{F}_{2}$, and $\mathcal{F}_{1}$. We have already observed that $\mathcal{F}_{1}^{1}$ and $2 \mathcal{F}_{2}^{2}$ represent trivial elements in $\mathcal{C}$. Thus, we are reduced to considering $4 \mathcal{F}_{4}^{\infty}, 2 \mathcal{F}_{2}^{\infty}, \mathcal{F}_{1}^{\infty}$ and $\mathcal{F}_{1}^{2}$. Here is the list of knots, with names now attached for later discussions.

- $4 \mathcal{F}_{4}^{\infty}: K_{1}=4\left(7_{7}\right)$

- $2 \mathcal{F}_{2}^{\infty}: K_{2}=2\left(8_{1}\right), K_{3}=2\left(8_{13}\right), K_{4}=2\left(8_{15}-7_{2}-3_{1}\right)$

- $\mathcal{F}_{1}^{\infty}: K_{5}=8_{21}-8_{18}-3_{1}$

- $\mathcal{F}_{1}^{2}: K_{6}=8_{17}-8_{17}^{r}$

Individual knots in this subgroup have been discussed in previous articles: that $7_{7}$ is of infinite order in $\mathcal{C}$ is a consequence of Casson-Gordon theory, as presented in [20]; the nontriviality of $8_{17}-8_{17}^{r}$ was among the first applications of the twisted Alexander polynomials viewed as a discriminant of a Casson-Gordon invariant, in [16. Twisted polynomials were used in 26] to prove that $2\left(8_{13}\right)$ is not slice, and further Casson-Gordon techniques were applied to show that $8_{1}$ is of infinite order. 
5.1. Twisted Alexander polynomials as slicing obstructions. Let $M_{q}(K)$ denote the $q$-fold branched cover of $S^{3}$ branched over $K$, where $q$ is a prime power. For each prime $p$ and homomorphism $\rho: H_{1}\left(M_{q}(K)\right) \rightarrow \mathbf{Z}_{p}$, there is a twisted Alexander polynomial, $\Delta_{K, \rho}(t) \in \mathbf{Q}\left[\zeta_{p}\right]\left[t, t^{-1}\right]$. In [15] this polynomial is shown to be related to a Casson-Gordon invariant of $K$. In particular, if $K$ is slice, then for certain $\rho$, it is shown that $\Delta_{K, \rho}(t)$ factors as $a f(t) \overline{f\left(t^{-1}\right)}$ for some $f(t) \in$ $\mathbf{Q}\left[\zeta_{p}\right]\left[t, t^{-1}\right]$, where $a$ is some unit in $\mathbf{Q}\left[\zeta_{p}\right]\left[t, t^{-1}\right]$.

Restricting the set of characters that one must consider is one of the most challenging aspects of the computations. In brief, there is a linking form on the first homology group of the branched cover and there is also a $\mathbf{Z}_{q}$ action on this group. The appropriate set of characters is given by linking with elements in a subgroup of the homology of the cover, and this subgroup should be invariant under the group action and be self-annihilating with respect to the linking form. Details can be found in [16].

The computation of $\Delta_{K, \rho}$ is completely algorithmic; in 14 methods were developed that ensured the rapid computer calculation of these polynomials, sufficient to work with all prime knots of 12 or fewer crossings. For any individual knot, one can often enumerate the possible $\rho$ that must be considered and carry out the computation. The problem of showing linear independence was considered in [17, where the methods were developed that could isolate properties of the necessary families of characters $\rho$; these methods were applied to families of knots specifically designed to be accessible by the new techniques.

5.2. The homology of the covers. Fortunately, for the knots of interest, a large number of primes occur in considering only the 2-fold and 3-fold branched covers. Here are the needed results.

- $\left|H_{1}\left(M_{2}\left(7_{7}\right)\right)\right|=3 \cdot 7$

- $\left|H_{1}\left(M_{2}\left(8_{1}\right)\right)\right|=13$

- $\left|H_{1}\left(M_{2}\left(8_{13}\right)\right)\right|=29$

- $\left|H_{1}\left(M_{2}\left(8_{15}\right)\right)\right|=3 \cdot 11$

- $\left|H_{1}\left(M_{2}\left(7_{2}\right)\right)\right|=11$

- $\left|H_{1}\left(M_{2}\left(3_{1}\right)\right)\right|=3$

- $\left|H_{1}\left(M_{2}\left(8_{21}\right)\right)\right|=3 \cdot 5$

- $\left|H_{1}\left(M_{2}\left(8_{18}\right)\right)\right|=3^{2} \cdot 5$

- $\left|H_{1}\left(M_{2}\left(8_{17}\right)\right)\right|=37 \quad\left|H_{1}\left(M_{3}\left(8_{17}\right)\right)\right|=13^{2}$

5.3. Summary calculation. Suppose a linear combination of the knots $a_{1} K_{1}+$ $a_{2} K_{2}+a_{3} K_{3}+a_{4} K_{4}+a_{5} K_{5}+a_{6} K_{6}=0 \in \mathcal{C}$. Working with the 2-fold covers and the primes 7,13 , and 29 lets one conclude that the coefficients $a_{1}, a_{2}$ and $a_{3}$ all are zero. Because $K_{6}=8_{17}-8_{17}^{r}$, it turns out that working with the prime 37 does not imply that $a_{6}=0$. However, this is the only knot in the list for which 13 appears in the 3 -fold cover, and as described in [16, this is sufficient to show that the coefficient $a_{6}=0$. (Actually, since $K_{6}$ is of order two, one shows only that $a_{6}$ is even.)

At this point, one need only consider combinations $a_{4} K_{4}+a_{5} K_{5}$. The primes 11 and 5 let one reduce this to considering each knot individually, for which another computation of twisted Alexander polynomials, along with detailed work at identifying possible metabolizers, completes the project. A few more details are presented in the next section. 


\section{Model ClassificAtion theOREM}

In this section we will illustrate the general procedure used to attain the eight crossing classification by carrying out the classification for a smaller subset of knots. These knots were chosen because their analysis entails most of the necessary steps while avoiding the lengthy computations. If knots have relatively prime Alexander polynomials, the work usually is simplified, so we have selected these knots to share polynomial factors. These examples skirt two difficult issues, reversibility and four torsion in $\mathcal{G}$. The first is dealt with in detail for the one relevant knot among eight crossing knots in [16]. With respect to four torsion, the algebraic issues are presented in [18, 19, 21]; the only relevant example is $7_{7}$, which is of infinite order in $\mathcal{C}$ and of algebraic order four, as discussed in detail in [20]. These examples also provide an opportunity to discuss two issues regarding numerical approximation that arise.

We consider the set consisting of the following six knots, listed with the homology of the 2 -fold branched cover, $H$, and the irreducible factorization of the Alexander polynomial, $\Delta$. We let the subgroup they generate in $\mathcal{C}$ be denoted $\mathcal{D}$.

- $3_{1}: H=\mathbf{Z}_{3} ; \Delta=1-t+t^{2}$.

- $7_{2}: H=\mathbf{Z}_{11} ; \Delta=3-5 t+3 t^{2}$.

- $8_{10}: H=\mathbf{Z}_{27} ; \Delta=\left(1-t+t^{2}\right)^{3}$.

- $8_{15}: H=\mathbf{Z}_{3} \oplus \mathbf{Z}_{11} ; \Delta=\left(1-t+t^{2}\right)\left(3-5 t+3 t^{2}\right)$.

- $8_{18}: H=\mathbf{Z}_{3} \oplus \mathbf{Z}_{3} \oplus \mathbf{Z}_{5} ; \Delta=\left(1-t+t^{2}\right)^{2}\left(1-3 t+t^{2}\right)$.

- $8_{21}: H=\mathbf{Z}_{3} \oplus \mathbf{Z}_{5} ; \Delta=\left(1-t+t^{2}\right)\left(1-3 t+t^{2}\right)$.

Infinite order elements in $\mathcal{G}$ : Of the three irreducible polynomials that appear in these factorizations, two, $1-t+t^{2}$ and $3-5 t+3 t^{2}$, have roots on the unit circle. Since these polynomials are quadratic, this is quickly checked, but we note it can also be determined numerically. The existence of a nonreal root close to the unit circle can be verified numerically. But for a symmetric real polynomial, if $\alpha$ is a nonreal root close to, but not on, the unit circle, then $1 / \bar{\alpha}$ is a distinct nearby root. Such a root could be approximated numerically; if it doesn't exist, then $\alpha$ is on the unit circle.

The two roots of the polynomials are approximately at $\alpha=e^{.333 \pi i}$ and $\beta=$ $e^{.186 \pi i}$. Thus we select the numbers $A=e^{.25 \pi i}$ and $B=e^{.5 \pi i}$ and compute the value of the signature functions at these points. Again we can work numerically; the signature function is defined for all complex numbers, not just those on the unit circle, and is constant on the domains complementary to the (real) lines through 1 and the roots of the unit circle. Thus, the values are stable under small perturbations away from these lines. The values of the signature function at $A$ and $B$ (denoted $\sigma_{A}$ and $\sigma_{B}$ ) are as follows.

- $3_{1}: \sigma_{A}=0, \sigma_{B}=-2$.

- $7_{2}: \sigma_{A}=-2, \sigma_{B}=-2$.

- $8_{10}: \sigma_{A}=0, \sigma_{B}=2$.

- $8_{15}: \sigma_{A}=-2, \sigma_{B}=-4$.

- $8_{18}: \sigma_{A}=0, \sigma_{B}=0$.

- $8_{21}: \sigma_{A}=0, \sigma_{B}=-2$. 
We now make a change of basis, forming a new set of six knots as follows.

- $3_{1}: \sigma_{A}=0, \sigma_{B}=-2$.

- $7_{2}: \sigma_{A}=-2, \sigma_{B}=-2$.

- $8_{18}: \sigma_{A}=0, \sigma_{B}=0$.

- $8_{10}+3_{1}: \sigma_{A}=0, \sigma_{B}=0$.

- $8_{15}-3_{1}-7_{2}: \sigma_{A}=0, \sigma_{B}=0$.

- $8_{21}-3_{1}: \sigma_{A}=0, \sigma_{B}=0$.

Thus, the first two knots on this list map to a free subgroup of rank two in $\mathcal{G}$ and the others split off a summand of $\mathcal{D}$ mapping to torsion in $\mathcal{G}$. We relist them with their Alexander polynomials.

- $8_{18}: H=\mathbf{Z}_{3} \oplus \mathbf{Z}_{3} \oplus \mathbf{Z}_{5} ; \Delta=\left(1-t+t^{2}\right)^{2}\left(1-3 t+t^{2}\right)$.

- $8_{10}+3_{1}: H=\mathbf{Z}_{27} \oplus \mathbf{Z}_{3} ; \Delta=\left(1-t+t^{2}\right)^{4}$.

- $8_{15}-3_{1}-7_{2}: H=\mathbf{Z}_{3} \oplus \mathbf{Z} \oplus \mathbf{Z}_{11} \oplus \mathbf{Z}_{11} ; \Delta=\left(1-t+t^{2}\right)^{2}\left(3-5 t+3 t^{2}\right)^{2}$.

- $8_{21}-3_{1}: H=\mathbf{Z}_{3} \oplus \mathbf{Z}_{3} \oplus \mathbf{Z}_{5} ; \Delta=\left(1-t+t^{2}\right)^{2}\left(1-3 t+t^{2}\right)$.

Since $8_{18}$ and $8_{21}-3_{1}$ have Alexander polynomials with the factor $1-3 t+t^{2}$ having odd exponent, they both map to elements of order two in $\mathcal{G}$. Thus, we perform a further change of basis, replacing $8_{21}-3_{1}$ with $8_{21}-8_{18}-3_{1}$ so that it now clearly represents either 0 or an element of order two distinct from $8_{18}$ in $\mathcal{G}$. Also, we note that $8_{18}$ is amphicheiral and so represents an element of order 2 in $\mathcal{C}$. We can also now use the fact that a simple concordance from $8_{10}$ to $-3_{1}$ has been constructed, so that $8_{10}+3_{1}$ represents 0 in $\mathcal{C}$. Thus, the problem is reduced to considering the following pair of knots.

- $J_{1}=8_{15}-3_{1}-7_{2}: H=\left(\mathbf{Z}_{3}\right)^{2} \oplus\left(\mathbf{Z}_{11}\right)^{2} ; \Delta=\left(1-t+t^{2}\right)^{2}\left(3-5 t+3 t^{2}\right)^{2}$.

- $J_{2}=8_{21}-8_{18}-3_{1}: H=\left(\mathbf{Z}_{3}\right)^{4} \oplus\left(\mathbf{Z}_{5}\right)^{2} ; \Delta=\left(1-t+t^{2}\right)^{4}\left(1-3 t+t^{2}\right)^{2}$.

These knots each represent elements of order at most two in $\mathcal{G}$. Whether they are trivial or not can be detected by considering the linking form on the 2-fold cover. To do this, one needs to consider the form defined by $\left(V+V^{t}\right)^{-1}$ on the first homology, where $V$ is a Seifert matrix. For the first knot, $J_{1}$, restricting to the 11 -torsion, the form is diagonal, with diagonal entries $\left(-\frac{1}{11}, \frac{2}{11}\right)$. Since 2 is not a square modulo 11, the form is not metabolic, and thus the knot represents a nontrivial element of order 2 in $\mathcal{G}$. On the other hand, for the second knot, $J_{2}$, all torsion invariants vanish; in particular, the linking form on the 5 -torsion is diagonalized as $\left(\frac{1}{5}, \frac{1}{5}\right)$, which is Witt trivial, since -1 is a square modulo 5 . Similar considerations based on Levine's computations show that $J_{2}$ is algebraically slice.

It remains to prove the independence of $J_{1}$ and $J_{2}$ in $\mathcal{C}$. This is done by considering twisted Alexander polynomials at the primes 5 and 11. For these knots it suffices to consider the $2-$ fold branched covers, $M_{2}(K)$, for each appropriate $K$. For any knot $K$ and a homomorphism $\chi: H_{1}\left(M_{2}(K)\right) \rightarrow \mathbf{Z}_{p}$ there is defined a twisted Alexander polynomial $\Delta_{K, \chi}(t) \in \mathbf{Q}\left[\zeta_{p}\right]\left[t, t^{-1}\right]$. If $K$ is slice, then for appropriate $\chi$ related to a metabolizer of the linking form on $M_{2}(K)$, these polynomials factor as norms, $a f(t) \overline{f\left(t^{-1}\right)}$ for some $f$.

Suppose that $a J_{1}+b J_{2}=0 \in \mathcal{C}$. Then $2 a J_{1}+2 b J_{2}=0$, and since $8_{18}$ is amphicheiral, by expanding $J_{1}$ and $J_{2}$ we see that $\left(2 a 8_{15}-2(a+b) 3_{1}-2 a 7_{2}\right)+$ $b 8_{21}=0$. Consider a character $\chi$ to $\mathbf{Z}_{5}$. Since the only one of these knots which has 
five torsion in the 2 -fold cover is $8_{21}$, we conclude that for appropriate constants,

$$
\Delta_{3_{1}, 0}(t)^{x} \Delta_{7_{2}, 0}(t)^{y} \Delta_{8_{15}, 0}(t)^{z} \Delta_{8_{21}, 0}(t)^{w} \Delta_{8_{21}, \chi}(t)^{m} \Delta_{8_{21}, 2 \chi}(t)^{n}
$$

is a norm. The factors $\Delta_{0}$ are twisted polynomials corresponding to the trivial representation and are determined by the Alexander polynomial. The twisted polynomials for characters $\chi$ and $2 \chi$ arise from the summands of the branched cover on which the given character agrees with $\pm \chi$ or with $\pm 2 \chi$, respectively. The exponents $x, y, z, w, m$, and $n$ are determined by a corresponding element in the metabolizer. For the two polynomials of greater interest, one computes:

$$
\begin{gathered}
\Delta_{8_{21}, \chi}(t)=t^{2}-3\left(\omega^{3}+\omega^{2}+1\right) t+1, \\
\Delta_{8_{21}, 2 \chi}=t^{2}-3\left(\omega^{4}+\omega+1\right) t+1 .
\end{gathered}
$$

(Notice that these polynomials are self-conjugate and are not conjugate to each other. For instance, their product is not a norm.)

At this point, one must undertake a technical analysis of possible metabolizers of the linking form. Here is a summary. The relevant linking form is defined on $\left(\mathbf{Z}_{5}\right)^{2 b}$. Any metabolizer is of rank $b$, generated by $b$ vectors in $\left(\mathbf{Z}_{5}\right)^{2 b}$. These vectors can be viewed as the rows of a $b \times 2 b$ matrix with entries in $\mathbf{Z}_{5}$. A change of basis permits one to write the matrix in the form $(I E)$ where $I$ is an identity matrix and $E$ is nonsingular. There is the following result, Lemma 5.14 of [17].

Proposition. Let $E$ be a nonsingular $k \times k$ matrix over $\mathbf{Z}_{p}$ for a prime $p>2$. Suppose that the subspace of $\left(\mathbf{Z}_{p}\right)^{2 k}$ spanned by the rows of the $k \times 2 k$ matrix $(I E)$ contains no odd vectors (that is, every vector in this span has an even number of nonzero entries). Then $E$ is obtained from a diagonal matrix by permuting the columns.

From this one can conclude that for some metabolizing element, in the corresponding twisted polynomial one of the exponents $m$ or $n$ is necessarily odd. For the given polynomials this implies the product of polynomials cannot be a norm, which it would be if the knot were slice.

It follows that $b=0$. A similar argument, using the prime 11, implies that $a=0$, but somewhat more care is required because the prime 11 is a factor of the orders of the homology of the 2 -fold covers of both $7_{2}$ and $8_{15}$.

Combining these observations, we summarize with the following.

Summary. Let $\mathcal{F}$ denote the free abelian group generated by the knots $3_{1}, 7_{2}, 8_{10}$, $8_{15}, 8_{18}$, and $8_{21}$. Then $\mathcal{F}$ splits into the direct sum of subgroups, with generators as indicated. The subscripts indicate the algebraic order of elements and the superscripts the concordance order.

- $\mathcal{F}_{\infty}^{\infty}: 3_{1}, 7_{2}$

- $\mathcal{F}_{2}^{\infty}: 8_{15}-7_{2}-3_{1}$

- $\mathcal{F}_{2}^{2}: 8_{18}$

- $\mathcal{F}_{1}^{\infty}: 8_{21}-8_{18}-3_{1}$

- $\mathcal{F}_{1}^{1}: 8_{10}+3_{1}$ 


\section{Appendix. Nine CROSSING KNOTS}

The analysis of the subgroup of $\mathcal{C}$ generated by prime knots of nine or fewer crossings was undertaken in the Ph.D. thesis of the first author, available at [3]. In this case there are 87 knots to consider, including three arising from nonreversible knots: $8_{17}^{r}, 9_{32}^{r}$ and $9_{33}^{r}$. There are again seven relevant subgroups of the free abelian group $\mathcal{F}$ generated by these 87 knots (with superscripts indicating their order in $\mathcal{C}$ and subscripts the order in $\mathcal{G}$ ):

- $\mathcal{F}_{\infty}^{\infty}: 3_{1}, 5_{1-2}, 6_{2}, 7_{1-6}, 8_{2}, 8_{4-7}, 8_{14}, 8_{16}, 8_{19}, 9_{1-2}, 9_{4-7}, 9_{9-11}, 9_{13}, 9_{15}$, $9_{17-18}, 9_{20-22}, 9_{25-26}, 9_{31-32}, 9_{35-36}, 9_{38}, 9_{43}, 9_{45}, 9_{47-49}$

- $\mathcal{F}_{4}^{\infty}: 7_{7}, 9_{34}$

- $\mathcal{F}_{2}^{\infty}: 8_{1}, 8_{13},\left(8_{15}-7_{2}-3_{1}\right),\left(\mathbf{9}_{\mathbf{2}}-\mathbf{7}_{\mathbf{4}}\right),\left(9_{12}-5_{2}\right), 9_{14},\left(9_{16}-7_{3}-3_{1}\right)$, $9_{19},\left(9_{28}-3_{1}\right), 9_{30}, 9_{33},\left(9_{42}+8_{5}-3_{1}\right),\left(9_{44}-4_{1}\right)$

- $\mathcal{F}_{2}^{2}: 4_{1}, 6_{3}, 8_{3}, 8_{12}, 8_{17-18}$

- $\mathcal{F}_{1}^{\infty}:\left(\mathbf{8}_{\mathbf{2 1}}-\mathbf{8}_{\mathbf{1 8}}-\mathbf{3}_{\mathbf{1}}\right),\left(9_{8}-8_{14}\right),\left(\mathbf{9}_{\mathbf{2 3}}-\mathbf{9}_{\mathbf{2}}-\mathbf{3}_{\mathbf{1}}\right),\left(9_{29}-9_{28}+2\left(3_{1}\right)\right)$, $\left(\mathbf{9}_{\mathbf{3 2}}^{\mathbf{r}}-\mathbf{9}_{\mathbf{3 2}}\right),\left(9_{33}^{r}-9_{33}\right),\left(9_{39}+7_{2}-4_{1}\right),\left(\mathbf{9}_{\mathbf{4 0}}-\mathbf{8}_{\mathbf{1 8}}-\mathbf{4}_{\mathbf{1}}-\mathbf{3}_{\mathbf{1}}\right)$

- $\mathcal{F}_{1}^{2}:\left(8_{17}^{r}-8_{17}\right)$

- $\mathcal{F}_{1}^{1}: 6_{1}, 8_{8-9},\left(8_{10}+3_{1}\right),\left(8_{11}-3_{1}\right), 8_{20},\left(9_{24}-4_{1}\right), 9_{27},\left(9_{37}-4_{1}\right), 9_{41}, 9_{46}$

The classification is nearly complete, with the exception that the span in $\mathcal{C}$ of the five knots shown in bold is not fully identified. Of these, the first is of algebraic order two and the rest are algebraically slice. Each of the individual knots is of infinite order in $\mathcal{C}$. However, in [3] and in all subsequent work there has been no success in ruling out any possible relations between these five, except for the fact that any such relation must contain an even multiple of the first.

Thus the concordance classification of nine crossing knots will be completed by answering the following question: For what integers $a_{1}, a_{2}, a_{3}, a_{4}, a_{5}$ is the linear combination

$$
2 a_{1} J_{1}+a_{2} J_{2}+a_{3} J_{3}+a_{4} J_{4}+a_{5} J_{5}
$$

slice, with

$$
\begin{gathered}
J_{1}=9_{2}-7_{4}, J_{2}=8_{21}-8_{18}-3_{1}, J_{3}=9_{23}-9_{2}-3_{1}, \\
J_{4}=9_{32}^{r}-9_{32}, J_{5}=9_{40}-8_{18}-4_{1}-3_{1} ?
\end{gathered}
$$

\section{REFERENCES}

[1] A. J. Casson and C. McA. Gordon, Cobordism of classical knots, With an appendix by P. M. Gilmer. À la recherche de la topologie perdue, Progr. Math., vol. 62, Birkhäuser Boston, Boston, MA, 1986, pp. 181-199. MR900252

[2] J. C. Cha and C. Livingston, KnotInfo: Table of Knot Invariants, www.indiana.edu / knotinfo, June 17, 2013.

[3] J. Collins, On the concordance orders of knots, arxiv preprint: arXiv.org/abs/1206.0669 .

[4] Tim D. Cochran and Robert E. Gompf, Applications of Donaldson's theorems to classical knot concordance, homology 3-spheres and property P, Topology 27 (1988), no. 4, 495-512, DOI 10.1016/0040-9383(88)90028-6. MR976591 (90g:57020)

[5] T. D. Cochran and W. B. R. Lickorish, Unknotting information from 4-manifolds, Trans. Amer. Math. Soc. 297 (1986), no. 1, 125-142, DOI 10.2307/2000460. MR849471 (87i:57003)

[6] Tim D. Cochran, Kent E. Orr, and Peter Teichner, Structure in the classical knot concordance group, Comment. Math. Helv. 79 (2004), no. 1, 105-123, DOI 10.1007/s00014-001-0793-6. MR2031301 (2004k:57005) 
[7] J. H. Conway, An enumeration of knots and links, and some of their algebraic properties, Computational Problems in Abstract Algebra (Proc. Conf., Oxford, 1967), Pergamon, Oxford, 1970, pp. 329-358. MR.0258014 (41 \#2661)

[8] Ronald Fintushel and Ronald J. Stern, Pseudofree orbifolds, Ann. of Math. (2) 122 (1985), no. 2, 335-364, DOI 10.2307/1971306. MR808222 (87a:57027)

[9] Ralph H. Fox and John W. Milnor, Singularities of 2-spheres in 4-space and cobordism of knots, Osaka J. Math. 3 (1966), 257-267. MR0211392 (35 \#2273)

[10] Michael H. Freedman and Frank Quinn, Topology of 4-manifolds, Princeton Mathematical Series, vol. 39, Princeton University Press, Princeton, NJ, 1990. MR.1201584 (94b:57021)

[11] Patrick M. Gilmer, Slice knots in $S^{3}$, Quart. J. Math. Oxford Ser. (2) 34 (1983), no. 135, 305-322, DOI 10.1093/qmath/34.3.305. MR711523(85d:57004)

[12] Joshua Greene and Stanislav Jabuka, The slice-ribbon conjecture for 3-stranded pretzel knots, Amer. J. Math. 133 (2011), no. 3, 555-580, DOI 10.1353/ajm.2011.0022. MR2808326 (2012e:57005)

[13] Matthew Hedden and Paul Kirk, Instantons, concordance, and Whitehead doubling, J. Differential Geom. 91 (2012), no. 2, 281-319. MR2971290

[14] Chris Herald, Paul Kirk, and Charles Livingston, Metabelian representations, twisted Alexander polynomials, knot slicing, and mutation, Math. Z. 265 (2010), no. 4, 925-949, DOI 10.1007/s00209-009-0548-1. MR2652542 (2011g:57006)

[15] Paul Kirk and Charles Livingston, Twisted Alexander invariants, Reidemeister torsion, and Casson-Gordon invariants, Topology 38 (1999), no. 3, 635-661, DOI 10.1016/S00409383(98)00039-1. MR:1670420(2000c:57010)

[16] Paul Kirk and Charles Livingston, Twisted knot polynomials: inversion, mutation and concordance, Topology 38 (1999), no. 3, 663-671, DOI 10.1016/S0040-9383(98)00040-8. MR 1670424 (2000c:57011)

[17] P. Kirk and C. Livingston, Concordance and mutation, Geom. Topol. 5 (2001), 831-883 (electronic), DOI 10.2140/gt.2001.5.831. MR.1871406 (2002j:57016)

[18] J. Levine, Invariants of knot cobordism, Invent. Math. 8 (1969), 98-110; addendum, ibid. 8 (1969), 355. MR0253348 (40 \#6563)

[19] Charles Livingston, The algebraic concordance order of a knot, J. Knot Theory Ramifications 19 (2010), no. 12, 1693-1711, DOI 10.1142/S0218216510008571. MR2755496 (2012e:57013)

[20] Charles Livingston and Swatee Naik, Obstructing four-torsion in the classical knot concordance group, J. Differential Geom. 51 (1999), no. 1, 1-12. MR.1703602 (2000g:57009)

[21] Toshiyuki Morita, Orders of knots in the algebraic knot cobordism group, Osaka J. Math. 25 (1988), no. 4, 859-864. MR983807 (90d:57028)

[22] D. D. Long, Strongly plus-amphicheiral knots are algebraically slice, Math. Proc. Cambridge Philos. Soc. 95 (1984), no. 2, 309-312, DOI 10.1017/S0305004100061569. MR.735371 (85h:57007)

[23] Kunio Murasugi, On a certain numerical invariant of link types, Trans. Amer. Math. Soc. 117 (1965), 387-422. MR0171275 (30 \#1506)

[24] Jacob Rasmussen, Khovanov homology and the slice genus, Invent. Math. 182 (2010), no. 2, 419-447, DOI 10.1007/s00222-010-0275-6. MR2729272 (2011k:57020)

[25] Lee Rudolph, Quasipositivity as an obstruction to sliceness, Bull. Amer. Math. Soc. (N.S.) 29 (1993), no. 1, 51-59, DOI 10.1090/S0273-0979-1993-00397-5. MR1193540 (94d:57028)

[26] Andrius Tamulis, Knots of ten or fewer crossings of algebraic order 2, J. Knot Theory Ramifications 11 (2002), no. 2, 211-222, DOI 10.1142/S0218216502001585. MR.1895371 (2003b:57012)

School of Mathematics and Maxwell Institute for Mathematical Sciences, UniverSity OF Edinburgh, Scotland, EH9 3JZ

E-mail address: Julia.Collins@ed.ac.uk

Department of Mathematics, Indiana University, Bloomington, Indiana 47405

E-mail address: pkirk@indiana.edu

Department of Mathematics, Indiana University, Bloomington, Indiana 47405

E-mail address: livingst@indiana.edu 